\title{
MSH2 Missense Mutations and HNPCC Syndrome: Pathogenicity Assessment in a Human Expression System
}

\author{
Laura Belvederesi ${ }^{1}$, Francesca Bianchi ${ }^{1}$, Eva Galizia ${ }^{1}$, Cristian Loretelli ${ }^{1}$, Raffaella Bracci ${ }^{1}$, \\ Romina Catalani ${ }^{1}$, Monica Amati ${ }^{2}$, and Riccardo Cellerino ${ }^{1}$ \\ ${ }^{1}$ Centro Regionale di Genetica Oncologica - Oncologia Medica, Università Politecnica delle Marche, Ancona \\ ${ }^{2}$ Dipartimento di Patologia Molecolare e Terapie Innovative, Università Politecnica delle Marche, Ancona
}

*Correspondence to: Laura Belvederesi, Ph.D, Centro Regionale di Genetica Oncologica - Oncologia Medica, Università Politecnica delle Marche, Via Tronto 10, 60126, Ancona, Italy;

E-mail: 1.belvederesi@univpm.it

Communicated by Riccardo Fodde

\begin{abstract}
Hereditary Non-Polyposis Colorectal Cancer (HNPCC) is associated with germline mutations in one of several MisMatch Repair (MMR) genes. An increasing proportion (20$25 \%$ ) of the reported MSH2 variants consists of single amino-acid substitution with uncertain disease-causing significance. The present study was undertaken to functionally characterize 3 MSH2 nontruncating variants: p.Gly162Arg (c.484G $>$ C), p.Asp167His $($ c.499G $>C$ C) and p.Arg359Ser $($ c.1077A $>$ T). Missense alterations, were assessed in a human system for expression/stability and for the ability to heterodimerize with MSH6 and correctly localize into the nucleus. Functional assays results were correlated with clinical and genetic features indicative of HNPCC as MicroSatellite-Instability (MSI), abnormalities of MMR gene expression in tumour tissue (IHC) and familial history. p.Gly162Arg and p.Arg359Ser variants showed a clearly decreased expression level of the MutSa complex and were associated with an abnormal subcellular localization pattern, which can be suggestive of an incorrect MSH2/MSH6 heterodimerization. Functional analysis results were supported by MSI and IHC data and by familial cancer history. The subcellular localization assay, performed in a human expression system, classifies as pathogenetic two MSH2 nontruncating alterations providing a useful tool in genetic testing programs. () 2008 WileyLiss, Inc.
\end{abstract}

KEY WORDS: HNPCC; MMR; MSH2; missense variants; functional analysis

\section{INTRODUCTION}

Hereditary Non-Polyposis Colorectal Cancer (HNPCC; MIM\# 120435) is a dominantly inherited cancer susceptibility syndrome which accounts for 3-5\% of all colorectal tumours (de la Chapelle 2005). HNPCC syndrome is characterized by an increasing lifetime risk of colorectal as well as of other cancers such as endometrium, stomach or small bowel and is genetically associated with germline mutations in one of several postreplicative DNA MisMatch Repair (MMR) genes, mostly MLH1 (MIM\# 120436), MSH2 (MIM\# 609309), and, in fewer cases, MSH6 (MIM\# 600678) or PMS2 (MIM\# 600259) (Lynch and de la Chapelle 2003). MMR

Received 15 May 2008; accepted revised manuscript 09 Jul 2008.

(C) 2008 WILEY-LISS, INC.

DOI: 10.1002/humu.20875 
reverses base to base mismatches and slippages in simple repeat sequences that escape the DNA polymerase proofreading activity (Kunkel and Erie 2005).

For successful accomplishment of the MMR activity, the MSH2 and MSH6 repair proteins have to heterodimerize to constitute the MutS $\alpha$ complex and correctly translocate from the cytoplasm into the nucleus through NLS-importin $\alpha / \beta$ shuttling mechanism (Iyer et al. 2006, Knudsen et al. 2007, Stewart 2007). Defects in MMR activity result in a mutator phenotype that leads to increase spontaneous somatic mutation rate, particularly in multi simple repeats non-expressed sequences (microsatellite) (Peltomaki 2003, Kunkel and Erie 2005). Nearly 500 different and likely pathogenetic germline alterations affecting mostly the MLH1 (50\%), MSH2 (39\%) and MSH6 (7\%) MMR genes are listed in the international HNPCC mutations database (Peltomaki and Vasen 2004). Although the majority of $\mathrm{MSH} 2$ associated mutations result in premature termination of translation, a significant and increasing proportion (20-25\%) of the reported $\mathrm{MSH} 2$ variants give rise to single amino acid substitution (Peltomaki and Vasen 2004, Ollila et al. 2006, Woods et al. 2007).

Disease-causing nontruncating mutations can be distinguished from non-pathogenic variants by evaluating some well defined criteria: co-segregation with the disease, low incidence of the alteration in the general healthy population, non conservative status of the amino acidic change, evolutionary conservation of the involved codon, and, in cases of suspected HNPCC, correlation with MicroSatellite Instability (MSI) and loss of MMR proteins in tumour tissue (Genuardi et al. 1999, Cravo et al. 2002). However, rare polymorphisms can result in a nonconservative amino-acid change and segregation studies are rarely feasible because of ethical issues, limitations of family size and/or unavailability of biological specimens (Trojan et al. 2002, Kondo et al. 2003). In addition, the biochemical significance of these alterations is often uncertain and loss of MMR gene expression and MSI could represent the result of somatic mutations or epigenetic silencing (Cravo et al. 2002, Nystrom-Lahti et al. 2002, Yuen et al. 2002). In such cases, the nature of the inherited MMR mutation and its role in colorectal cancer susceptibility should be further analysed by other means.

In order to provide informations about the effect of the HNPCC-related gene variants on the function of the corresponding MMR polypeptide, several functional assays have been developed (Shimodaira et al. 1999, Guerrette et al. 1999, Shcherbakova and Kunkel 1999 Ellison et al. 2001, Brieger et al. 2002, Nyström-Lahti et al. 2002, Trojan et al. 2002, Kondo et al. 2003, Ellison et al. 2004, Raevaara et al. 2005, Belvederesi et al. 2006, Blasi et al. 2006, Takahashi et al 2007).

Contrary to MLH1 missense mutations, which have already been deeply assessed, there is a poor knowledge about the pathogenicity of MSH2 non truncating variants. Nearly all the already known MSH2 related assays are based on a biochemical approach (Kijas et al. 2003, Heinen et al. 2002) or conducted in a heterologous system (Ellison et al. 2001, Gammie et al. 2007). To our knowledge, only one recent study (Ollila et al. 2006) aimed to functionally characterise MSH2 missense variants in a human expression system and to date no pathogenic assessment dealing with MSH2 protein localization defects have been established.

The present study was undertaken to investigate, in a human expression system, the effects of $3 \mathrm{MSH} 2$ nontruncating variants, found in suspected HNPCC families, on subcellular localization of the corresponding protein and successively to validate such assay as a tool for MSH2 pathogenic assessment. To this aim, we both compared localization assay results to those obtained by less innovative approaches (expression/interaction assays) and correlated such results with in silico predictive alignment sequence analysis and with clinical data, such as familial cancer history, occurrence of MSI and abnormalities of MMR gene expression in the tumour tissue.

\section{PATIENTS AND METHODS}

\section{HNPCC families and MSH2 mutations}

Among the clinically characterised missense mutations identified in patients referred to our Centre for genetic counselling, only the ones for which functional data were available in the literature were included in the present study. The 3 MSH2 mutations were identified in 5 patients belonging to 3 unrelated HNPCC families. All the index patients had a family history fulfilling at least one of the Bethesda guidelines (Rodriguez-Bigas et al. 1997, Umar et al. 2004) and subscribed written informed consent to genetic testing and to the additional studies required for this work.

Mutational analysis was performed on genomic DNA purified from peripheral mononuclear blood cells, according to standard procedures. All the $M L H 1$ and $M S H 2$ exons, including flanking intronic regions, were 


\section{E298 Belvederesi et al.}

individually amplified and directly sequenced using Applied Biosystems ABI PRISM 310 automated sequencer and the Big Dye terminator Cycle Sequencing Ready Reaction-Kit (Applied Biosystems, Foster City, CA) as previously described (Scartozzi et al. 2002). Identified mutations were confirmed on a second sample PCR product. Primer sequences are available from the corresponding Author upon request.

The prevalence of each variant was evaluated in the germline of a reference population panel of 100 healthy blood donors and compared with the international HNPCC database (http://www.insight-group.org). Multiplex Ligation-dependent Probe Amplification (MLPA) was applied to exclude large genomic rearrangements and performed as previously described (Bianchi et al. 2007). A 40-55\% decrease of the area of an MSH2 or MLH1 exon peak compared to the wild-type control samples was considered as indicative of a heterozygous deletion of that exon.

\section{MSI and Immunohistochemical Analyses}

Colorectal cancer DNA was investigated for MSI using the 5-markers panel (two mononucleotide repeats BAT25 and BAT26- and three dinucleotide repeats -D2S123, D5S346, D17S250) recommended by international guidelines (Boland et al. 1998).

Microsatellite sequences were PCR amplified from tumour and matched normal DNA using 5'- fluorochrome labelled oligonucleotide primer pairs (Belvederesi et al. 2006). PCR products were analysed by capillary gel electrophoresis (ABI 310 Genetic Analyzer -Applied Biosystems) followed by automated allele sizing using the GeneScan 3.7 software (Applied Biosystems, Foster City, CA). PCR primers and conditions are available from the corresponding Author. Tumours were classified as highly unstable (MSI-H) when instability was found in at least $40 \%$ of the analysed microsatellite markers. ImmunoHistoChemical (IHC) analysis of MLH1, MSH2 and MSH6 protein expression was performed on $2 \mu \mathrm{m}$ sections of paraffin-embedded tumour tissue samples, following antigen retrieval (Marcus et al. 1999). The following primary antibodies and dilutions were used: anti-MLH1 protein (clone G168-728, PharMingen, San Diego, CA), 1:50 dilution; anti-MSH2 protein (clone FE11, Calbiochem/Oncogene Research Products, Cambridge, MA), 1:100 dilution; anti-MSH6 protein (clone H-141, Santa Cruz Biotechnology, Santa Cruz, CA) 1:250 dilution.

\section{Comparative sequence analysis}

The Sorting Intolerant From Tolerant (SIFT) software (http://blocks.fhcrc.org/sift/SIFT html) was adopted to predict the effects of MSH2 amino-acid substitutions on protein functions (Ng and Henikoff 2003). SIFT algorithm, through alignments of homologue and evolutionary conserved sequences, calculates normalized probabilities for all possible substitutions from the alignments and classifies all potential amino-acid variants as tolerated or deleterious. Positions with normalized probabilities less than .05 are predicted to be deleterious, those greater than or equal to .05 are predicted to be tolerated ( $\mathrm{Ng}$ and Henikoff 2003). Program default settings were used.

\section{Cloning of $\mathbf{M S H} 2$ and $\mathbf{M S H} 6$ genes}

The entire MSH2 (GenBank NM_000251.1) and MSH6 (GenBank NM_000179.2) encoding regions were RTPCR amplified from the MMR-competent 293 human cell line, using engineered primers bearing the BamHI XhoI restriction sites. (Primer sequences and PCR conditions are available from the corresponding Author). The resulting PCR products were directionally cloned into the pCAN-TAGs eucariotic expression vector, a modification of pcDNA3 (Invitrogen, Carlsbad, CA) containing an oligoaminoacidic myc-Tag upstream the Multiple Cloning Site (MCS). The in frame expression of the myc-Tag and the amino terminus of MSH2 or MSH6 generates the myc-MSH2 and myc-MSH6 tagged fusion protein (recombinant constructs were referred as pCANmyc-MSH2 and pCAN-myc-MSH6). MSH2 cDNA was also subcloned between the same restriction sites of pGEX4T-1 (Amersham Bioscience, Piscataway, NJ) bacterial expression vector to generate the Glutathione-STransferase-MSH2 fusion protein GST-MSH2 (pGEX-MSH2). The whole Open Reading Frame (ORF) of MSH2 and of MSH6, including the insertion sites in the expression vectors, were verified by direct DNA sequencing. 


\section{Site direct mutagenesis and vectors production}

A PCR based site-directed mutagenesis method (Nystrom-Lahti et al. 2002) was used to reproduce the three MSH2 missense mutations found in our HNPCC patients: MSH2(p.G162R), MSH2(p.D167H) and MSH2(p.R359S). Two further variants, MSH2(p.P622L) and MSH2(c.2633_2634delAG, p.Glu878fsX3), thought to compromise the correct subcellular localization and to impair the MSH2/MSH6 heterodimerization respectively (Knudsen et al. 2007, Heinen et al. 2002), were generated. All variants numbering is based on MSH2 cDNA sequence (GenBank NM_000251.1) and uses the major translation initiation site as codon 1.

PCR products containing the mutation were directionally ligated between the BamHI and HindIII cloning sites into the template construct pGEX-MSH2 replacing the corresponding wild-type (WT) region. The following constructs were obtained: pGEX-GST-MSH2(p.G162R), pGEX-GST-MSH2(p.D167H) and pGEX-GSTMSH2(p.R359S).

For protein expression in human LoVo cells, the resulting MSH2 mutant derivatives were subcloned into the pCAN-TAGs expression vector between BamHI and XhoI restriction sites to obtain pCAN-myc-MSH2(p.G162R), pCAN-myc-MSH2(p.D167H) and pCAN-myc-MSH2(p.R359S). The introduction of the specific mutation and the absence of undesired second site sequence alterations were confirmed by sequence analysis. PCR primers and conditions are available from the corresponding Author.

For fluorescent protein production, the WT MSH2 cDNA was cloned into the pEGFP-N1 vector (BD Biosciences, Palo Alto,CA) between the XhoI and BamHI restriction sites and in frame with the amino terminus of the Enhanced Green Fluorescent Protein (EGFP) encoding gene. The resulting construct expressing MSH2-EGFP fluorescent fusion protein is reported here as pEGFP-MSH2. The mutant MSH2 derivatives pEGFPMSH2(p.G162R), pEGFP-MSH2(p.D167H) and pEGFP-MSH2(p.R359S) were generated to the pEGFP-MSH2 plasmid between the XhoI and HindIII sites by site-directed mutagenesis as described above.

\section{Expression of MSH2 and MSH6 in LoVo and 293 cells}

In order to assess the MutS $\alpha$ proteins expression levels in a suitable homologue system, a total of $2 \mathrm{X} 10^{6}$ MMR-deficient LoVo adenocarcinoma cells were transiently co-transfected with $1 \mu \mathrm{g}$ of pCAN-myc-MSH2 (WT or mutant) and pCAN-myc-MSH6 recombinant expression vectors using LipofectAMIN ${ }^{\mathrm{TM}} 2000$ transfection reagent (Invitrogen) as specified by the manufacturer. pCAN-TAGs vector without insert was transfected as mock control.

For total protein extraction, cells were harvested $48 \mathrm{~h}$ after transfection and lysed into cold extraction buffer (Belvederesi et al. 2006). Equal amounts $(60 \mu \mathrm{g})$ of the whole cell lysate were resolved by $6 \%$ SDS-PAGE and electroblotted to a PVDF membrane (Millipore, Bedford, MA). The expression levels and correct sizes of MutS $\alpha$ recombinant proteins, myc-MSH2 and myc-MSH6, were assessed by Western immunoblotting using a mouse monoclonal primary antibody (Clone 9E10, 1:600 dilution, Santa Cruz Biotechnology) specific for the myc-Tag of the fusion proteins.

Amounts of 6 X $10^{6}$ MMR proficient 293 human cells were transiently transfected with $6 \mu \mathrm{g}$ of purified pCANmyc-MSH6 vector mixed with $40 \mu$ of LipofectAMIN ${ }^{\mathrm{TM}} 2000$ (Invitrogen). Cells were lysed $48 \mathrm{~h}$ after transfection and the effective ectopic over-expression of the myc-Tag fused MSH6 protein was verified by SDS-PAGE and Western immunoblot analysis using the anti-myc-Tag primary antibody as described above. As endogenous MSH6 was weakly expressed in 293 cells (data not shown), pooled 293 transfected cells lysates were used as a source of WT MSH6 protein for the MSH2/MSH6 interaction assay as described in the following paragraph.

\section{GST fusion protein interaction assay}

Wild-type and site-directly mutated MSH2 cDNAs were cloned into pGEX4T-1 vector and expressed into competent Escherichia coli BL21 cells as GST-fused MSH2 proteins. Recombinant MSH2 proteins were purified using a GST pull-down method as previously described (Guerrette et al. 1998). Wild type MSH6 was cloned into pCAN-TAGs vector and retrieved as ectopic over-expressed form from 293 (MMR+) lysates.

Amounts of BL21 total extracts evaluated to contain equal quantities of recombinant MSH2 (about 800 ng) were pre-bound to glutathione-agarose beads and incubated for $2 \mathrm{~h}$ with 293 cell lysate expressing wild type mycMSH6, in $500 \mu \mathrm{l}$ of binding buffer (Belvederesi et al. 2006). Agarose beads-bound proteins were successively resolved by $6 \%$ SDS-PAGE. The interaction of MSH2 variants with their biochemical partner MSH6 to constitute 
the MutS $\alpha$ heterodimer was detected by Western immunoblotting analysis with a mouse monoclonal antibody (dilution 1:600) against the MSH6 myc-tag (Clone 9E10, Santa Cruz Biotechnology). In order to confirm that equal amounts of WT or mutant GST-MSH2 proteins were used in the assay, the same PVDF membrane was subsequently probed with a monoclonal antibody to the MSH2 protein (clone FE11, 1:100 dilution, Calbiochem/Oncogene Research Products).

\section{Subcellular localization analysis}

For the expression of WT or mutant EGFP-MSH2 fluorescent fusion proteins in 293 cells, the transfection procedure was similar to as previously described except that $0.5 \times 10^{6} 293$ cells were seeded onto polylysine treated $(0.1 \mathrm{mg} / \mathrm{ml})$ glass coverslips and culture medium was replaced 6 hours after transfection.

For fluorescent EGFP-MSH2 fusion proteins detection, the 293 cells were fixed 24 hours after transfection with a paraformaldehyde solution (4\% in PBS, $\mathrm{pH} 7.4$ ) for 20 minutes at room temperature and stained with a $1 \mu \mathrm{g} / \mathrm{ml}$ solution of the DNA intercalating specific dye 4',6-DiAmidino-2-PhenylIndole (DAPI) (Sigma Aldrich, St Louis, MO). Subcellular localization of EGFP-MSH2 recombinant proteins was analysed by direct fluorescence using an Imager A1 microscope with a 63X objective (Zeiss, Thornwood, NY). Representative images were captured with Axio Cam MRC5 and processed with Axio Vision software (Zeiss). Images were representative for at least $80 \%$ of the transfected cells analyzed. At least 100 cells, from each of the three individual transfection we performed, were analyzed from randomly sampled microscope fields of view.

\section{RESULTS}

\section{Patients, genetic studies and tumour phenotypes}

Three germline MSH2 missense mutations were identified in 5 patients belonging to 3 unrelated families who underwent genetic testing for suspected HNPCC (Table 1). The mutations were c.484G $>$ C (p.Gly162Arg), c.499G $>$ C (p.Asp167His) and c.1077A $>$ T (p.Arg359Ser). All variants numbering is based on MSH2 cDNA sequence (GenBank NM_000251.1) and uses the A of the ATG translation initiation start site as nucleotide +1 .

These genetic variants affected conserved amino-acid residues causing non conservative amino-acid changes ( $\mathrm{Ng}$ and Henikoff 2003), were not listed among the known MSH2 polymorphisms reported in inSiGHT database and were not found in a control group of 100 healthy volunteers. Furthermore MLPA analysis did not show any evidence of large genomic rearrangements in $\mathrm{MLH} 1$ and $\mathrm{MSH} 2$ genes.

The p.G162R mutation was found in a 55 years old patient who developed two synchronous colorectal cancers and whose family history complied with the Bethesda criteria ( 2 guidelines). The assessment of the MSI showed instability of 3 out of the 5 microsatellite markers. IHC showed normal MLH1 expression and loss of MSH2 and MSH6 protein expression. The p.D167H mutation was found in a patient who was diagnosed with an adenocarcinoma of the right colon at 42 years of age, without any significant family history of HNPCC-related tumours (Scartozzi et al 2002). IHC showed loss of MLH1 protein expression whereas the immuno-expression of MSH2 and MSH6 was normal. MSI and MLPA analysis were inconclusive because of the low quantity of germline DNA and tumour tissue specimens. The p.R359S mutation was found in 3 related patients whose family history fulfilled the Amsterdam II criteria showing a typical HNPCC pedigree (Bianchi et al. 2007). Index patient MSI analysis resulted in a high degree of instability (80\%). Normal staining for MLH1 and the lack of MSH2 and MSH6 expression were observed in the tumour tissue. MLPA analysis did not show other germline abnormalities of MLH1 and MSH2 which could be responsible of the strong HNPCC phenotype of this patient. Phenotypic characteristics of the variants included in the present investigation are listed in Table 1. 
Table 1. Clinical, genetic and functional features associated with the three MSH2 missense variants.

\begin{tabular}{|c|c|c|c|c|c|c|c|c|c|c|c|c|}
\hline Age & $\begin{array}{l}\text { Family } \\
\text { history }\end{array}$ & $\begin{array}{c}A A \\
\text { change }\end{array}$ & $\begin{array}{c}\text { Type } \\
\text { AA change }\end{array}$ & SIFT & MSI & $\begin{array}{c}\text { IHC } \\
\text { MSH2 }\end{array}$ & $\begin{array}{c}\text { IHC } \\
\text { MSH6 }\end{array}$ & $\begin{array}{c}\text { IHC } \\
\text { MLH1 }\end{array}$ & Expression & $\begin{array}{l}\text { Interaction } \\
\text { MSH2/MSH6 }\end{array}$ & Localization & $\begin{array}{l}\text { Relationship } \\
\text { with HNPCC }\end{array}$ \\
\hline 55 & Beth. & p.G162R & $\begin{array}{c}\text { Nonpolar } \rightarrow \\
\text { Basic }\end{array}$ & D & $60 \%$ & - & - & + & Reduced & + & $\begin{array}{c}\text { Altered } \\
\text { (nucleus+cytoplasm) }\end{array}$ & Likely \\
\hline 42 & Beth. & p.D167H & $\begin{array}{l}\text { Acidic } \rightarrow \\
\text { Basic }\end{array}$ & D & ND & + & + & - & Normal & + & $\begin{array}{c}\text { Normal } \\
\text { (nucleus) }\end{array}$ & Unlikely \\
\hline 59 & Amst. II & p.R359S & $\begin{array}{l}\text { Basic } \rightarrow \\
\text { Uncharged } \\
\text { polar }\end{array}$ & D & $80 \%$ & - & - & + & Reduced & + & $\begin{array}{c}\text { Altered } \\
\text { (cytoplasm) }\end{array}$ & Likely \\
\hline
\end{tabular}

Sequence variants numbering is based on MSH2 cDNA sequence (GenBank NM_000251.1) and uses the ATG translation initiation site as codon 1. NOTE: Amst., patients fulfilling the Amsterdam Criteria; Beth., patients fulfilling at least one of the Bethesda Guidelines; D, amino-acidic substitution predicted deleterious (score $\leq .05$ ) by SIFT software; ND, not diagnostic; WT, wild type; MSI, percentage of the microsatellite markers showing instability.

\section{Function - structure relationship}

Comparative sequence analysis system (SIFT software) was used to predict the outcome of the three MSH2 missense variants under study, utilizing the standard alignment and a cut-off score of .05 . Each of the three assessed variants was reliably (median conservation value less than 3.25) classified as deleterious by SIFT with a score next to 0.00 . Two out of the three variant sorted as intolerant showed functional defects suggesting a good accuracy for pathogenicity prediction.

\section{Expression of MSH2 and MSH6 in LoVo human cells}

An accurately regulated steady state expression level of the MSH2/MSH6 repair proteins is required for successful MutS $\alpha$ heterodimerization and accomplishment of the MMR activity. As in absence of its biochemical partner MSH2, endogenous MSH6 results unstable and undergoes to a post-translational downregulation of the cell level (Chang et al. 2000, Brieger et al. 2002), the effects of the MSH2 missense variant on protein stability were studied in LoVo human expression system following transient co-transfection with wild type MSH6.

Anti-myc Western blotting analysis (Figure 1) compares the MSH2-mutated protein expression levels with the MSH2-WT ones in co-transfected LoVo cells. Co-transfection with pCAN-myc-MSH2(p.D167H) resulted in a myc-MSH2 protein expression level similar to that referred to MSH2-WT, whereas a clearly decreased expression level of myc-MSH2 and myc-MSH6 proteins was detected co-transfecting pCAN-myc-MSH6 with pCAN-mycMSH2(p.G162R) or pCAN-myc-MSH2(p.R359S), suggesting that these MSH2 variants were rather unstable and/or unable to completely bind and stabilize MSH6 in vivo. Expression level reflecting amounts of MSH2 similar or lower than the WT were respectively classified as normal or reduced (Table 1). 


\section{E302 Belvederesi et al.}

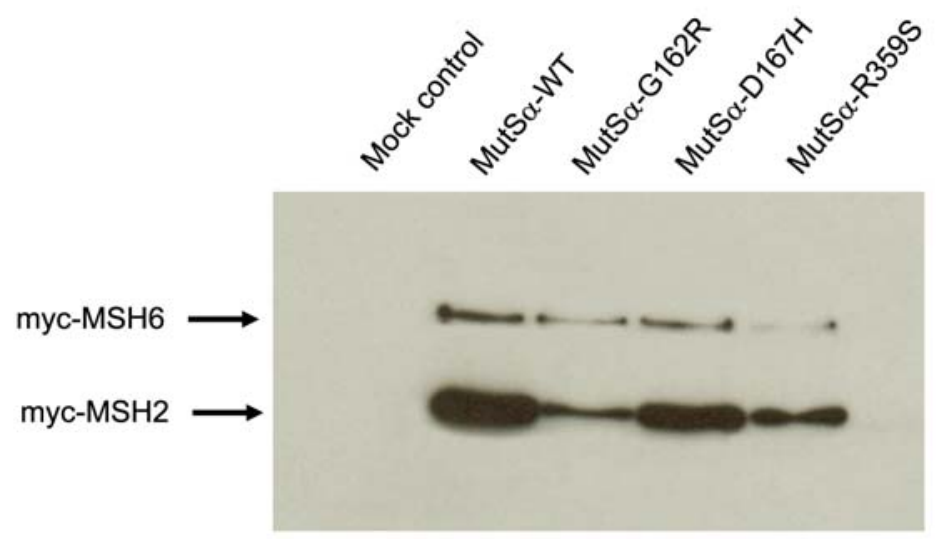

Figure 1. Analysis of expression levels of recombinant MSH2 variants coexpressed with MSH6 in human LoVo cells. Extracts were prepared 48h after co-transfection of LoVo cells with pCAN-myc-MSH2, wild-type or mutant, and pCAN-myc-MSH6 vectors. Equal amounts of total cell extract were resolved on 6\% SDS-PAGE and analysed by western immunoblotting using a monoclonal anti-myc antibody to detect the expression of myc-MSH2 and myc-MSH6 fusion protein. Mock control: cell lysate transfected with empty pCAN-TAGs expression vector.

\section{MSH2-MSH6 in vitro interaction assay}

The MSH2 protein exists mainly as a complex with MSH6, known as MutS $\alpha$, which is essential for MMR activity. The inability of HNPCC-linked MSH2 variants to interact with MSH6 could result in loss of MMR functionality and led to cancer susceptibility.

Although the 3 missense mutations we found did not occur within the two MSH2/MSH6 interaction domain (Guerrette et al. 1998), an alteration of the tridimensional protein conformation is not excluded. In order to investigate the effect of the three HNPCC related MSH2 missense mutations on MSH2/MSH6 binding impairment, we performed a combined GST-pull-down assay and anti-myc Western immunoblotting analysis. These experiments showed that none of the three MSH2 missense mutations MSH2(p.G162R), MSH2(p.D167H) and MSH2(p.R359S) yielded polypeptides that were unable to interact with their cognate partner MSH6. The cterm truncating MSH2 mutation (p.Glu878fsX3), thought to be able to impair such MSH2/MSH6 interaction, reduced but did not completely abolish MutS $\alpha$ assembly (Figure 2a).

In order to ascertain that an equivalent amount of each GST-MSH2 mutant was used in the interaction assay, the immunoblotted membranes were re-analysed with an anti-MSH2 antibody. As shown in Figure $2 \mathrm{~b}$, both MSH2-WT and each of the four MSH2 variants, including the truncating one, expressed comparable amounts of recombinant GST-tagged MSH2 polypeptides. No expression is detectable in the lane referred to the mock control (pGEX4T-1 vector without MSH2 cDNA). 


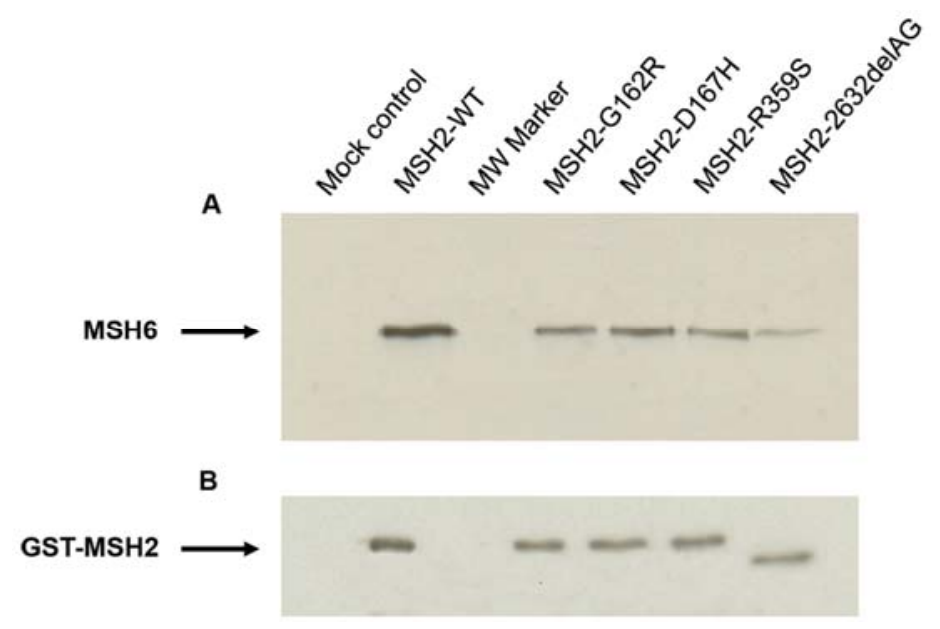

Figure 2. MSH2 germ-line mutations effect on MSH2-MSH6 heterodimerization. (a) In vitro interaction between WT or recombinant MSH2 variants and WT MSH6. Purified GST-MSH2 proteins, expressed in E. coli bacterial system, were incubated with a 293 total cell lysate expressing myc-MSH6. Assembly of the GST-MSH2/myc-MSH6 complex was detected by western immunoblotting with a monoclonal antibody to the myc-tag (b) Amounts of wild-type or mutant GST-MSH2 protein used in the MSH2/MSH6 interaction test. The PVDF membrane used to study the assembly of the MSH2/MSH6 complex (a) was probed with a monoclonal antibody to the MSH2 protein. Mock control: bacterial lysate transformed with empty pGEX4T-1 vector.

\section{Subcellular localization assay}

Defects in subcellular localization of the repair proteins result in a MMR-deficient phenotype, making nuclear import of MMR components a limiting step for the MMR repairing functions (Wu et al. 2003, Brieger et al. 2005, Knudsen et al. 2007).

As endogenous 293 MSH6 expression level was sufficient to drive the correct nuclear import of the MSH2/MSH6 preformed cytoplasmic MutS $\alpha$ complex (data not shown), location of WT and mutated EGFPMSH2 fluorescent fusion proteins was analysed in 293 human cells by simple transfection. Localization analysis in the MMR deficient LoVo cell line was not feasible as it resulted in cellular suffering and apoptosis related to the re-establishment of a defective MMR (data not shown).

The EGFP-MSH2(WT) fusion protein was correctly imported through the Nuclear Pore Complex (NPC) and detected almost completely in the nuclear district (Figure 3a), whereas the EGFP-MSH2(p.P622L) one, known as a nuclear import impairing variant (Knudsen et al.2007), coexpressed with endogenous MSH6, was mainly located in the cytoplasm and showed a diffuse nuclear-cytoplasmic distribution pattern (Figure3b). The EGFPMSH2(p.D167H) variant, whose nuclear proportion was similar to the EGFP-MSH2(WT) one, was classified as normal in the localization study (Figure 3d). The EGFP-MSH2(p.G162R) and EGFP-MSH2(p.R359S) variants were associated with an abnormal subcellular localization pattern of the corresponding fusion protein (Figure $3 \mathrm{c}$ and 3e). The first one (p.G162R) was evenly spread out among nuclear and cytoplasmic compartments whereas the other one (p.R359S) was mostly located in the cytoplasm, showing a defective nuclear localization as the dark fluorescenceless nuclear shape underlines. The results of the localization study are listed in Table1 and shown in Figure 3. 
E304 Belvederesi et al.
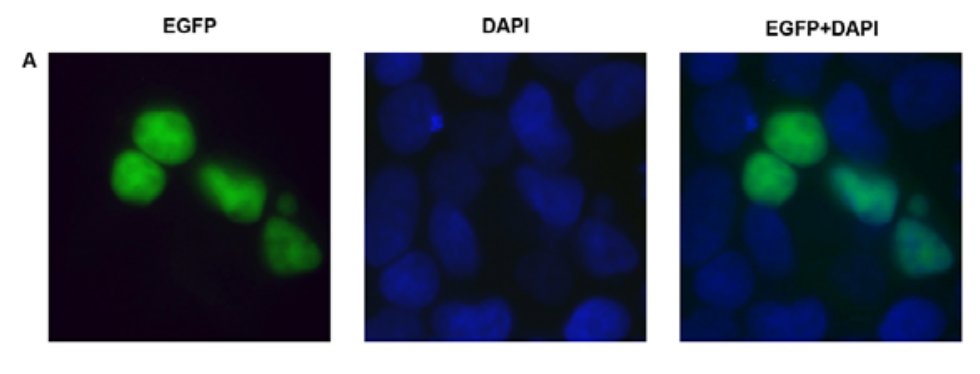

EGFP-MSH2 WT
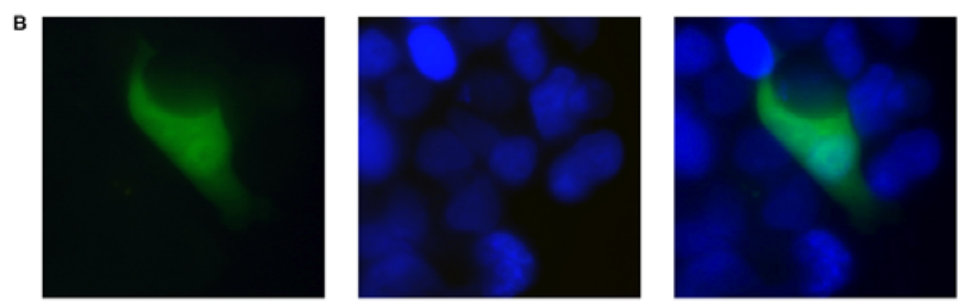

EGFP-MSH2 P622L
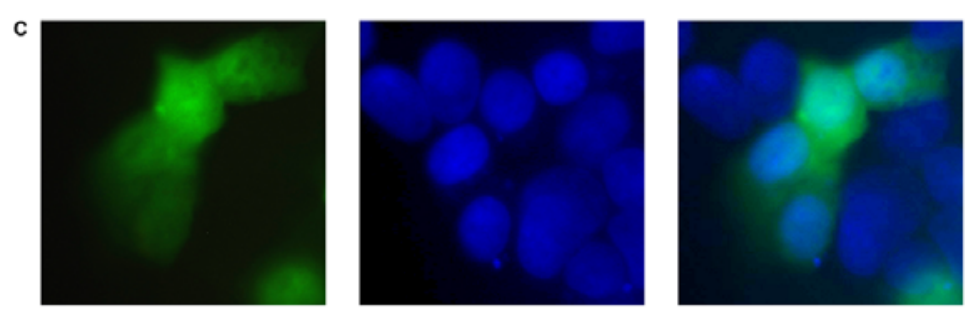

EGFP-MSH2 G162R
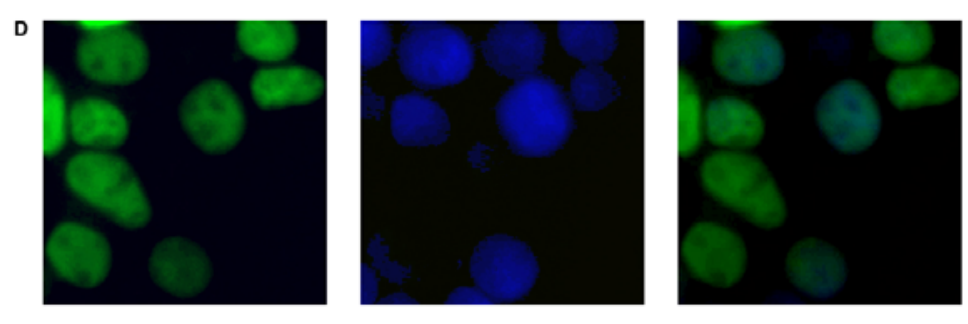

EGFP-MSH2 D167H
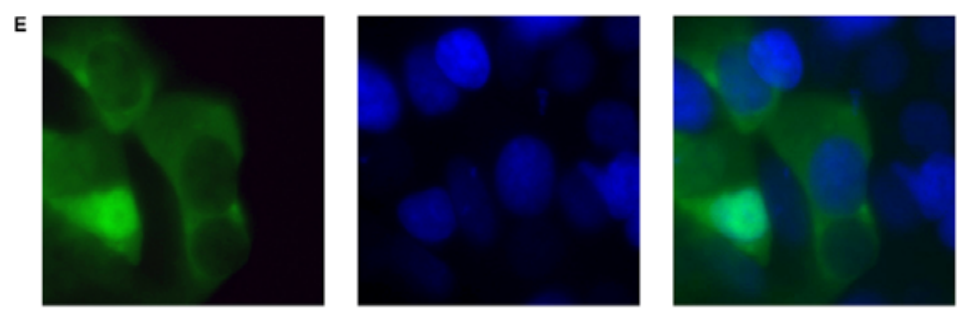

Figure 3: Localization analysis of recombinant wild type and mutated EGFP-MSH2 fusion proteins transiently expressed in 293 MMR proficient cells. Nuclei were stained with 4',6-diamino-2-phenylindole ( DAPI) and detected by direct fluorescence analysis $24 \mathrm{~h}$ after transfection. a)Nuclear expression of EGFP-MSH2(WT) (normal localization ctrl) b)Cytoplasmic expression of EGFP-MSH2(P622L) (abnormal localization ctrl) c)Mainly cytoplasmic expression of EGFP-MSH2(G162R) mutation similar to abnormal localization ctrl d)Nuclear expression of EGFP-MSH2(D167H) variant similar to EGFP-MSH2WT e)Exclusively cytoplasmic location of EGFP-MSH2(R359S) mutation which hinders the nuclear import. (original magnification $630 \mathrm{X})$ 


\section{DISCUSSION}

HNPCC is an hereditary form of colorectal cancer associated with germline deleterious defects in postreplicative DNA MMR genes, mainly MLH1, MSH2 and MSH6 (de la Chapelle 2004, Peltomaki 2005).

The effectiveness of HNPCC genetic testing deals with the identification of healthy carriers who can be counselled regarding the risk of colorectal cancer and enrolled in surveillance programmes. For a clinically useful genetic counselling it is necessary to ascertain the nature of the inherited MMR genes germline mutation and the role played in cancer risk. Such characterization, unambiguous in case of mutations that result in premature truncated proteins, can be more difficult for nontruncating variants.

A significant fraction (20-25\%) of HNPCC related $\mathrm{MSH} 2$ germline mutations deals with single aminoacid changes often associated with atypical clinical phenotypes (Peltomaki and Vasen 2004, Ollila et al. 2006). When linkage analysis are not feasible and the biochemical significance of the alteration is uncertain, the nature of the mutation should be functionally characterized before confidently assigning a pathogenetic role.

As functional characterization of MSH2 missense variants can be considered a developing area of interest, the present study attempt to contribute to this trend analyzing 3 nontruncating MSH2 mutations, found in suspected HNPCC families, by a rather new approach.

The 3 missense mutations we studied shared significant genetic similarities: they affected conserved amino acid residues, caused non conservative amino acid changes, were not reported among the known polymorphisms listed in the inSiGHT database and were absent in a control panel of 100 healthy individuals. Although these features are typical of deleterious mutations, their HNPCC causative role was to be ascertained by functional tools. Germline alterations were analyzed in a homologous human MMR system by investigating distinct functional properties mainly associated with stability and localization of the mutated proteins. To date and according to our knowledge, MSH2 missense mutations functional analysis dealing with the alteration of the subcellular localization are not available in the literature.

First of all, we evaluated the MSH2 and MSH6 steady-state protein levels to determine the effects of MSH2 missense mutations on protein stability. As previously showed by other Authors (Raevaara et al. 2005), to effectively detect decreases in protein quantity and obtain reliable data about protein stability, a sensitive expression system is required. Thus, recombinant protein variants were transiently co-expressed in human MSH2deficient LoVo cells. Two (p.G162R and p.R359S) out of the three variants were expressed in significantly lower amounts than the wild type protein, suggesting that these aminoacid replacements indirectly affect MMR efficiency through the alteration of the mutant protein stability. Both the variants resulting in decreased protein levels deal with substitutions of amino acids involved in protein folding. In particular, Glycine distorts $\alpha$-helical structures whereas Arginine, given the larger dimension, shows a propensity to be surface exposed (Pacios 2001, Duclohier 2004). Thus, as misfolded variants are often targeted for degradation (Goldberg 2003), it is not surprising that a single amino acid change, which interfere with normal protein structure, could affect the stability of the gene product and result in a reduced expression of the protein.

This finding was supported by the IHC analysis, which showed, for both mutations, the loss of MSH2 protein in tumour tissues. The fact that variants associated with reduced in vitro expression of the corresponding protein show a complete loss of MSH2 in IHC analyses can simply be related to differences in protein abundance in vivo and in vitro.

All the mutations analysed retained the MSH2/MSH6 heterodimerizing ability in our GST pull-down assay. This is not surprising, as none of the variants was located in the MSH6-interaction region. However, even the complete deletion of the c-terminal interaction domain, linked to the p.Glu878fsX3 mutation used as a control, did not abolish the interacting ability. This finding, explained by the fact that MSH2 exhibits 2 distinct MSH6 interaction regions (codons 378-625 and 875-934) (Guerrette et al. 1998), makes the reliability and usefulness of pulldown-based assays methods questionable. Such uncertainty is proved by Ollila's co-immunoprecipitation assay results, according to which all the variants, included those located in the interaction regions, maintained the heterodimerizing ability (Ollila et al. 2006).

To date, MSH2 missense variants functional consequences, included the heterodimer formation, were mainly studied by yeast based assays (Ellison et al. 2001, Gammie et al. 2007). Such assays rely on the expression of a particular phenotype in a heterologous system which shares a significant but not complete rate of homology with the human one (Shcherbakova and Kunkel 1999, Shimodaira et al. 1999, Trojan et al. 2002). As it is formally possible that missense mutations might be deleterious only in certain genetic contexts (Heck et al. 2006), the 


\section{E306 Belvederesi et al.}

designation as pathogenic for these mutations could be inaccurate and the yeast system could prove to be an inadequate model for human functions analysis.

In order to overcome the hurdles, both in the use of heterologous yeast systems and in the reliability of pulldown assays, we set up an alternative approach analyzing the subcellular localization of the mutated variants in a human expression system. In such approach, the localization assay acquires a double extent: provides hints about the ability of the mutated protein to correctly move into the nucleus and can be indirectly considered as an informative test about the MSH2/MSH6 heterodimerizing skill of the variants. Indeed, although able to form complexes with the nuclear import factors importin $\alpha 3 / \beta$, up to date MSH2 protein is considered not to contain any classical Nuclear Localization Signal (NLS) sequences. It is possible that this MMR protein enters the nucleus hooked by its heterodimerizing partners, primarily MSH6. Indeed, MSH6 exhibits several aminoacidic clusters, peculiar to typical NLS, which are utilized to actively drive the preformed cytoplasmic MutS $\alpha$ complex into the nucleus (Christmann and Kaina 2000, Knudsen et al. 2007). As expected, the wild type MSH2 protein was detected almost completely in the nucleus, whereas p.P622L variant, already predicted to be pathogenetic (Heinen et al. 2002, Gammie et al. 2007, Knudsen et al. 2007), showed a reduced nuclear proportion when compared to the WT form. In this reliable grading system, two out of the three variants analysed (p.G162R and p.R359S) were associated with an altered subcellular localization, which can be suggestive of an incorrect MSH2/MSH6 heterodimerization that affects the nuclear import of MutS $\alpha$ complex.

A further pathogenicity assessment of the variants under study was achieved by complementing expression/localization assays results with computational data. The SIFT software predicted the functional results for 2 out of 3 variants (p.G162R and p.R359S), whereas for one amino acid substitution (p.D167H) the alignmentbased prediction and functional analysis gave conflicting results, accordingly to the estimated percentage of failure in correct prediction (Chan et al. 2007, Takahashi et al. 2007).

The localization analysis classification of the investigated variants as either pathogenic or non-pathogenic was supported by clinical and genetic features indicative of HNPCC, such as family cancer history, occurrence of MSI and MMR gene expression abnormalities. Mutations shown to cause severe defects in our localization assay (p.G162R and p.R359S) were mirrored by phenotypic characteristics of typical HNPCC, including strong family history of colon cancer, high MSI and loss of MSH2/MSH6 expression in tumour tissues. Moreover, consistent with our views, Gammie's and Ollila's recent works, through a yeast-based functional assay and a repair efficiency assessment respectively, assigned to the same variants a HNPCC causative role (Ollila et al. 2006, Gammie et al. 2007). On the contrary, the p.D167H mutation, functionally classified as a likely harmless variant, was detected in a young patient belonging to a mild colorectal cancer family and was associated with a negative immuno-staining for MLH1 protein (Scartozzi et al. 2002). Since no mutation was found in MLH1 gene, the immunohistochemical result could be hypothetically related to a somatic inactivation occurred during the tumorigenesis or to epigenetic silencing (promoter hypermethylation) frequent in sporadic colorectal cancer. Such a hypothesis would suggest a further analysis of the MLH1 promoter methylation status. Consistent with our views, recently published data also indicate this mutation as non-pathogenetic (Gammie et al. 2007). In the light of such considerations, proband's cancer development was probably not to be ascribed to the MSH2 missense variant, which can be presumably considered a rare polymorphism.

In conclusion, the subcellular localization assay, performed in a human homologous system, reliably classifies as pathogenetic two MSH2 nontruncating alterations (p.G162R and p.R359S) providing a useful tool for MSH2 variants pathogenicity assessment. Our findings also illustrate the importance of combining genetic and clinical observations with data from functional assays to state with confidence whether a missense mutation is likely to be the causative factor for cancer development in HNPCC families. Elucidation of the missense mutations pathogenetic role may increase the effectiveness of genetic testing programs and facilitate the planning of appropriate preventive strategies of high risk mutation carriers from family segregating such mutations.

\section{REFERENCES}

Belvederesi L, Bianchi F, Loretelli C, Gagliardini D, Galizia E, Bracci R, Rosati S, Bearzi I, Viel A, Cellerino R, Porfidi E. 2006. Assessing the pathogenicity of MLH1 missense mutations in patients with suspected hereditary nonpolyposis colorectal cancer: correlation with clinical, genetic and functional features. Eur J Hum Genet; 14(7):853-9.

Bianchi F, Galizia E, Porfiri E, Belvederesi L, Catalani R, Loretelli C, Bracci R, Bearzi I, Turchi C, Viel A, Cellerino R. 2007. A missense germline mutation in exon 7 of the MSH2 gene in a HNPCC family from center-Italy. Fam Cancer; 6(1):97-102. 
Blasi MF, Ventura I, Aquilina G, Degan P, Bertario L, Bassi C, Radice P, Bignami M. 2006. A human cell-based assay to evaluate the effects of alterations in the MLH1 mismatch repair gene. Cancer Res; 66(18):9036-44.

Boland CR, Thibodeau SN, Hamilton SR, Sidransky D, Eshleman JR, Burt RW, Meltzer SJ, Rodriguez-Bigas MA, Fodde R, Ranzani GN, Srivastava S. 1998. A National Cancer Institute Workshop on Microsatellite Instability for cancer detection and familial predisposition: development of international criteria for the determination of microsatellite instability in colorectal cancer. Cancer Res; 58(22):5248-57.

Brieger A, Trojan J, Raedle J, Plotz G, Zeuzem S. 2002. Transient mismatch repair gene transfection for functional analysis of genetic hMLH1 and hMSH2 variants. Gut; 51(5):677-84.

Brieger A, Plotz G, Raedle J, Weber N, Baum W, Caspary WF, Zeuzem S, Trojan J. 2005. Characterization of the nuclear import of human MutL $\alpha$. Mol Carcinog; 43(1):51-8.

Chan PA, Duraisamy S, Miller PJ, Newell JA, McBride C, Bond JP, Raevaara T, Ollila S, Nyström M, Grimm AJ, Christodoulou J, Oetting WS, Greenblatt MS. 2007. Interpreting missense variants: comparing computational methods in human disease genes CDKN2A, MLH1, MSH2, MECP2, and tyrosinase (TYR). Hum Mutat. Jul;28(7):683-93.

Chang DK, Ricciardiello L, Goel A, Chang CL, Boland CR. 2000. Steady-state regulation of the human DNA mismatch repair system J Biol Chem; 275(37):29178.

Christmann M, Kaina B. 2000. Nuclear translocation of mismatch repair proteins MSH2 and MSH6 as a response of cells to alkylating agents. J Biol Chem. 17;275(46):36256-62.

Cravo M, Afonso AJ, Lage P, Albuquerque C, Maia L, Lacerda C, Fidalgo P, Chaves P, Cruz C, Nobre-Leitão C. 2002. Pathogenicity of missense and splice site mutations in hMSH2 and hMLH1 mismatch repair genes: implications for genetic testing. Gut; 50(3):405-12.

de la Chapelle A. 2004. Genetic predisposition to colorectal cancer. Nat Rev Cancer; 4(10):769-80.

de la Chapelle A. 2005. The incidence of Lynch syndrome. Fam Cancer; 4(3):233-237.

Duclohier H. 2004. Helical kink and channel behaviour: a comparative study with the peptaibols alamethicin, trichotoxin and antiamoebin. Eur Biophys J; 33(3):169-74.

Ellison AR, Lofing J, Bitter GA. 2001. Functional analysis of human MLH1 and MSH2 missense variants and hybrid humanyeast MLH1 proteins in Saccharomyces cerevisiae. Hum Mol Genet; 10(18):1889-900.

Ellison AR, Lofing J, Bitter GA. 2004. Human MutL homolog (MLH1) function in DNA mismatch repair: a prospective screen for missense mutations in the ATPase domain. Nucleic Acids Res; 32(18):5321-38.

Gammie AE, Erdeniz N, Beaver J, Devlin B, Nanji A, Rose MD. 2007. Functional characterization of pathogenic human MSH2 missense mutations in Saccharomyces cerevisiae. Genetics; 177(2):707-21.

Genuardi M, Carrara S, Anti M, Ponz de Leòn M, Viel A. 1999. Assessment of pathogenicity criteria for constitutional missense mutations of the hereditary nonpolyposis colorectal cancer genes MLH1 and MSH2. Eur J Hum Genet; 7(7):77882.

Goldberg AL. 2003. Protein degradation and protection against misfolded or damaged proteins. Nature; 426(6968):895-9.

Guerrette S, Acharya S, Fishel R. 1999. The interaction of the human MutL homologues in hereditary nonpolyposis colon cancer. J Biol Chem; 274(10):6336-41.

Guerrette S, Wilson T, Gradia S, Fishel R. 1998. Interactions of human hMSH2 with hMSH3 and hMSH2 with hMSH6: examination of mutations found in hereditary nonpolyposis colorectal cancer. Mol Cell Biol; 18(11):6616-23.

Heck JA, Argueso JL, Gemici Z, Reeves RG, Bernard A, Aquadro CF, Alani E. 2006. Negative epistasis between natural variants of the Saccharomyces cerevisiae MLH1 and PMS1 genes results in a defect in mismatch repair. Proc Natl Acad Sci U S A; 103(9):3256-61.

Heinen CD, Wilson T, Mazurek A, Berardini M, Butz C, Fishel R. 2002. HNPCC mutations in hMSH2 result in reduced hMSH2-hMSH6 molecular switch functions. Cancer Cell; 1(5):469-78. 


\section{E308 Belvederesi et al.}

Iyer RR, Pluciennik A, Burdett V, Modrich PL. 2006. DNA mismatch repair: functions and mechanisms.Chem Rev;106(2):302-23.

Kijas AW, Studamire B, Alani E. 2003. Msh2 separation of function mutations confer defects in the initiation steps of mismatch repair. J Mol Biol; 331(1):123-38.

Knudsen NØ, Nielsen FC, Vinther L, Bertelsen R, Holten-Andersen S, Liberti SE, Hofstra R, Kooi K, Rasmussen LJ. 2007. Nuclear localization of human DNA mismatch repair protein exonuclease 1 (hEXO1). Nucleic Acids Res; 35(8):2609-19.

Kondo E, Suzuki H, Horii A, Fukushige S. 2003. A yeast two-hybrid assay provides a simple way to evaluate the vast majority of hMLH1 germ-line mutations. Cancer Res; 63(12):3302-8.

Kunkel TA, Erie DA. 2005. DNA mismatch repair. Annu Rev Biochem; 74:681-710.

Lynch HT, de la Chapelle. 2003. Hereditary colorectal cancer. N Engl J Med; 348(10):919-32.

Marcus VA, Madlensky L, Gryfe R, Kim H, So K, Millar A, Temple LK, Hsieh E, Hiruki T, Narod S, Bapat BV, Gallinger S, Redston M. 1999. Immunohistochemistry for hMLH1 and hMSH2: a practical test for DNA mismatch repair-deficient tumors. Am J Surg Pathol; 23(10):1248-55.

Ng PC, Henikoff S. 2003. SIFT: Predicting amino acid changes that affect protein function. Nucleic Acids Res; 31(13):3812-4.

Nyström-Lahti M, Perrera C, Räschle M, Panyushkina-Seiler E, Marra G, Curci A, Quaresima B, Costanzo F, D'Urso M, Venuta S, Jiricny J. 2002. Functional analysis of MLH1 mutations linked to hereditary nonpolyposis colon cancer. Genes Chromosomes Cancer; 33(2):160-7.

Ollila S, Sarantaus L, Kariola R, Chan P, Hampel H, Holinski-Feder E, Macrae F, Kohonen-Corish M, Gerdes AM, Peltomäki P, Mangold E, de la Chapelle A, Greenblatt M, Nyström M. 2006. Pathogenicity of MSH2 missense mutations is typically associated with impaired repair capability of the mutated protein. Gastroenterology; 131(5):1408-17.

Pacios LF. 2001. Distinct molecular surfaces and hydrophobicity of amino acid residues in proteins. J Chem Inf Comput Sci; 41(5):1427-35.

Peltomäki P. 2003. Role of DNA mismatch repair defects in the pathogenesis of human cancer. J Clin Oncol; 21(6):1174-9.

Peltomäki P, Vasen H. 2004. Mutations associated with HNPCC predisposition -- Update of ICG-HNPCC/INSiGHT mutation database. Dis Markers; 20(4-5):269-76.

Peltomäki P. 2005. Lynch syndrome genes. Fam Cancer; 4(3):227-32.

Raevaara TE, Korhonen MK, Lohi H, Hampel H, Lynch E, Lönnqvist KE, Holinski-Feder E, Sutter C, McKinnon W, Duraisamy S, Gerdes AM, Peltomäki P, Kohonen-Ccorish M, Mangold E, Macrae F, Greenblatt M, de la Chapelle A, Nyström M. 2005. Functional significance and clinical phenotype of nontruncating mismatch repair variants of MLH1. Gastroenterology; 129(2):537-49.

Rodriguez-Bigas MA, Boland CR, Hamilton SR, Henson DE, Jass JR, Khan PM, Lynch H, Perucho M, Smyrk T, Sobin L, Srivastava S. 1997. A National Cancer Institute Workshop on Hereditary Nonpolyposis Colorectal Cancer Syndrome: meeting highlights and Bethesda guidelines. J Natl Cancer Inst; 89(23):1758-62.

Scartozzi M, Bianchi F, Rosati S, Galizia E, Antolini A, Loretelli C, Piga A, Bearzi I, Cellerino R, Porfiri E. 2002. Mutations of hMLH1 and hMSH2 in patients with suspected hereditary nonpolyposis colorectal cancer: correlation with microsatellite instability and abnormalities of mismatch repair protein expression. J Clin Oncol; 20(5):1203-8.

Shcherbakova PV, Kunkel TA. 1999. Mutator phenotypes conferred by MLH1 overexpression and by heterozygosity for mlh1 mutations.Mol Cell Biol; 19(4):3177-83.

Shimodaira H, Filosi N, Shibata H, Suzuki T, Radice P, Kanamaru R, Friend SH, Kolodner RD, Ishioka C. 1999. Functional analysis of human MLH1 mutations in Saccharomyces cerevisiae. Nat Genet; 21(2):241.

Stewart M. 2007. Molecular mechanism of the nuclear protein import cycle. Nat Rev Mol Cell Biol; 8(3):195-208.

Takahashi M, Shimodaira H, Andreutti-Zaugg C, Iggo R, Kolodner RD, Ishioka C. 2007. Functional analysis of human MLH1 variants using yeast and in vitro mismatch repair assays. Cancer Res; 67(10):4595-604. 
Trojan J, Zeuzem S, Randolph A, Hemmerle C, Brieger A, Raedle J, Plotz G, Jiricny J, Marra G. 2002. Functional analysis of hMLH1 variants and HNPCC-related mutations using a human expression system. Gastroenterology; 122(1):211-9.

Umar A, Boland CR, Terdiman JP, Syngal S, de la Chapelle A, Rüschoff J, Fishel R, Lindor NM, Burgart LJ, Hamelin R, Hamilton SR, Hiatt RA, Jass J, Lindblom A, Lynch HT, Peltomaki P, Ramsey SD, Rodriguez-Bigas MA, Vasen HF, Hawk ET, Barrett JC, Freedman AN, Srivastava S. 2004. Revised Bethesda Guidelines for hereditary nonpolyposis colorectal cancer (Lynch syndrome) and microsatellite instability. J Natl Cancer Inst; 96(4):261-8.

Woods MO, Williams P, Careen A, Edwards L, Bartlett S, McLaughlin JR, Younghusband HB. 2007. A new variant database for mismatch repair genes associated with Lynch syndrome. Hum Mutat. Jul;28(7):669-73.

Wu X, Platt JL, Cascalho M. 2003. Dimerization of MLH1 and PMS2 limits nuclear localization of MutLalpha. Mol Cell Biol; 23(9):3320-8.

Yuen ST, Chan TL, Ho JW, Chan AS, Chung LP, Lam PW, Tse CW, Wyllie AH, Leung SY. 2002. Germline, somatic and epigenetic events underlying mismatch repair deficiency in colorectal and HNPCC-related cancers. Oncogene; 21(49):758592. 